\title{
Mieux comprendre l'émergence de nouvelles langues
}

Le rôle de la convergence dans l'évolution de la marque du parfait (f)in/'n dans certains créoles français

For a better understanding of the emergence of new languages - the role of convergence in the evolution of the perfect marker (f)in/' $n$ in some French creoles

\section{Sibylle Kriegel}

\section{(2) OpenEdition}

\section{Journals}

Édition électronique

URL : https://journals.openedition.org/tipa/4365

DOI : $10.4000 /$ tipa.4365

ISSN : 2264-7082

Éditeur

Laboratoire Parole et Langage

Référence électronique

Sibylle Kriegel, « Mieux comprendre l'émergence de nouvelles langues », TIPA. Travaux interdisciplinaires sur la parole et le langage [En ligne], 37 | 2021, mis en ligne le 23 juillet 2021, consulté le 20 octobre 2021. URL : http://journals.openedition.org/tipa/4365 ; DOI : https://doi.org/10.4000/tipa.4365

Ce document a été généré automatiquement le 20 octobre 2021.

\section{cc)}

La revue TIPA. Travaux interdisciplinaires sur la parole et le langage est mise à disposition selon les termes de la licence Creative Commons Attribution - Pas d'Utilisation Commerciale - Pas de Modification 4.0 International. 


\section{Mieux comprendre l'émergence de nouvelles langues}

Le rôle de la convergence dans l'évolution de la marque du parfait (f)in/'n dans certains créoles français

For a better understanding of the emergence of new languages - the role of convergence in the evolution of the perfect marker (f)in/'n in some French creoles

Sibylle Kriegel

\section{Histoire du concept : De la romanistique traditionnelle aux études créoles}

1 Hugo Schuchardt, dans son cours magistral initial à l'Université de Leipzig en 1870 discute de la classification possible des dialectes romans. Les dialectes, une fois qu'ils sont arrivés à l'âge d'homme - c'est la métaphore qu'utilise Schuchardt - prennent des évolutions en parallèle, sont même peut-être sujets à la convergence ('dann tritt parallele Entwicklung ein, ja vielleicht Konvergenz'). Bien évidemment, cet usage reste peu spécifique. Weinreich (1953), sans employer le terme de convergence aborde différents exemples de ce qu'il appelle interférences grammaticales (grammatical interference) pour conclure que le contact entre langues peut aboutir à une modification du type structurel de la langue en question. Language contact can result in such far reaching changes that the affected language assumes a different structural type. (Weinreich, 1953:42)

2 Ce constat de Weinreich forme le point de départ de l'article phare de Gumperz \& Wilson (1971) qui concluent qu'il n'existe pas de distinction a priori entre pidginisation, créolisation et d'autres processus de diffusion.

There seems to be no reason to draw an a priori distinction among pidginization, creolization and other diffusion processes; the difference may be merely one of degree. (Gumperz \& Wilson, $1971: 251$ ) 
Gumperz \& Wilson (1971) considèrent le rôle de la convergence dans le contact entre langues génétiquement non apparentées parlées dans le village indien de Kupwar. Ils se focalisent sur l'emploi des variétés locales du kannada (langue dravidienne), du marathi (langue indo-aryenne) et de l'ourdou (langue indo-aryenne) en étudiant un corpus de conversations naturelles d'interactions entre différents groupes sociaux, bilingues depuis plusieurs siècles. Ils montrent que, suite à des situations sociolinguistiques particulières, l'organisation syntaxique de ces langues se rapproche dans des processus de contact complexes. Ils étudient des cas de changement convergent (convergent change) de plusieurs traits morphosyntaxiques en montrant qu'un trait (p.ex. l'expression du genre) dans une langue (p.ex. le kupwar-ourdou) et dans une autre langue (p.ex. le kupwar-marathi) change pour ressembler de plus en plus au même trait dans une troisième langue (p.ex. le kupwar-kannada). Ils insistent sur la complexité des processus impliqués qui dépendent de conditions particulières variées, une approche que l'on peut qualifier d'écologique au sens de Ludwig et al. (2019). Gumperz \& Wilson (1971) travaillent avec des faisceaux de traits alors que la présente étude se limitera à présenter un trait individuel, la marque du parfait (f)in/'n dans les créoles mauricien et seychellois.

4 La première utilisation du mot convergence en études créoles remonte à Goodman (1964) qui étudie les origines de différents phénomènes morphosyntaxiques dans les créoles français en retraçant les possibles influences des dialectes français et des langues ouest-africaines. Dans son étude, il emploie le terme de convergence sans le définir, dans son explication de l'article postposé '-la' dans différents créoles français. Il postule l'existence d'une convergence entre le déterminant démonstratif français et l'article défini éwé provoquée par la quasi-identité phonétique entre les deux.

The Creole definite article and its variety of forms and functions would then seem to be due to a convergence of the French demonstrative and the Ewe definite article, no doubt conditioned by their virtual phonetic identity. (Goodman, 1964:

48)

5 En études créoles, c'est Bollée (1982) qui est la première à proposer une définition précise du terme en se fondant essentiellement sur les recherches concernant la diachronie des langues romanes. Selon elle,

La convergence fait référence au fait que des structures similaires présentes dans deux langues en contact se rencontrent et se renforcent mutuellement ou qu'une tendance observable dans une langue se grammaticalise sous l'influence d'une structure présente dans une autre langue. (Bollée, 1982 : 70, traduction SK)

6 Elle prend comme point de départ des études concernant l'évolution du français à partir du latin qui retracent des influences du superstrat germanique. Hilty $(1975: 413)$ p.ex. affirme que le superstrat francique a influencé des phénomènes déjà existants en gallo-roman de manière à les soutenir, les ralentir ou les accélérer (traduction SK). Tout en insistant sur les différences entre romanisation et créolisation, Bollée transpose les questions posées par Hilty à l'émergence des créoles français :

Doit-il exister un point d'ancrage ou de rattachement pour le transfert d'une construction non française? Ou serait-il également envisageable que des structures morphosyntaxiques qui sont entièrement étrangères au français puissent prendre le dessus? (Bollée, 1982 : 392, ma traduction)

7 Bien qu'il soit le premier en date, l'article de Bollée n'est pas le seul à se pencher sur la notion de convergence en études créoles. Il convient de mentionner Kihm (1988) qui propose un concept proche qu'il appelle conflation. Dans ses travaux, Chaudenson (p.ex. 
2003 : 404s.) souligne à plusieurs reprises l'importance de la convergence et Mufwene (p.ex. 2001 et 2008), dans le cadre de son approche de la compétition et de la sélection parle de congruence (voir ci-dessous). Baptista et al. (2016) examinent des effets de convergence avec une expérimentation psycholinguistique. Elles établissent une distinction entre transfert, convergence aréale et « convergence isomorphique ». Leur définition de la « convergence isomorphique » insiste sur la similarité entre les langues en contact. L'observation d'une similarité entre structures des langues en contact est également au cœur de l'approche proposée dans Kriegel et al. (2019) qui s'appuie sur les réflexions de Bollée (1982).

\section{En continuant les réflexions de Bollée : vers une nouvelle approche}

\subsection{Similarité et grammaticalisation}

Deux points dans la définition de Bollée forment le point de départ des propositions présentées dans Kriegel et al. (2019) :

9 La similarité entre deux structures dans deux langues : alors que Bollée se contente de constater l'existence de structures similaires, nous nous focalisons sur les locuteurs, les créateurs des langues créoles. Quand, dans une situation de communication marquée par le contact linguistique entre les langues (ou codes) A et B, des apprenants constatent une similarité entre un trait existant dans A, leur langue de départ, et $B$ qu'ils sont en train d'acquérir (le français parlé par les colons en l'occurrence), ils retiennent ce trait. Afin de conceptualiser cet acte de reconnaissance d'une similarité, nous nous fondons sur Jarvis \& Pavlenko (2008) qui parlent de perceived similarity. Les similarités perçues par des linguistes peuvent mais ne doivent pas obligatoirement coïncider avec les similarités perçues par les locuteurs, les apprenants.

A perceived similarity is a conscious or unconscious judgment that a form, structure, meaning, function, or pattern that an L2 user has encountered in the input of the recipient language is similar to a corresponding feature of the source language. (Jarvis \& Pavlenko, $2008: 179$ )

Dans notre approche, une condition sine qua non pour le déclenchement de processus de convergence entre des traits de deux (ou de plusieurs) langues en contact est la perception d'une similarité entre ces traits par les locuteurs. On retrouve cette idée déjà chez Weinreich (1953: 39) quand il constate que la motivation d'un bilingue à établir des équivalences entre morphèmes ou catégories de langues en contact est leur similarité formelle ou une similarité de fonctions pré-existante.

What leads the bilingual to establish the interlingual equivalence of the morphemes or categories is either their FORMAL SIMILARITY or a SIMILARITY IN PREEXISTING FUNCTIONS.

(Weinreich, 1953 : 39)

Le deuxième point important dans la définition de Bollée est novateur par rapport aux travaux précédents. Bollée parle bien d'une tendance observable dans une langue qui se grammaticalise sous l'influence d'une structure présente dans une autre langue. Dans notre approche de la convergence, la notion de grammaticalisation, traditionnellement utilisée dans un cadre monolingue (voir p.ex. Lehmann, 1995) est élargie aux situations écologiques plurilingues dans lesquelles évoluent les créoles (voir p.ex. Bruyn, 2009; Heine \& Kuteva, 2005 ; Kriegel 2003). ${ }^{1}$ 
12 Kriegel et al. (2019) insistent sur le rôle décisif des dialectes français impliqués dans la créolisation, une piste de recherche déjà initiée par les travaux de Chaudenson (p.ex. 2003). Dans la présente contribution, je souhaite davantage mettre l'accent sur le rôle qu'ont joué les langues des populations serviles dans la grammaticalisation d'une périphrase verbale présente en français à partir $d u 17^{\mathrm{e}}$ siècle. Ainsi, dans l'étude de cas présentée en 3., nous verrons qu'un processus de grammaticalisation "amorcé » en français (B) a été, sous l'influence substratique A1 (malgache) ainsi que sous l'influence substratique A2 (langues bantoues de l'Est), renforcé et accéléré dans les créoles mauricien et seychellois naissants (C). En addition aux propositions faites dans Kriegel et al. (2019), je propose d'appeler les créoles C afin d'insister sur le fait qu'il s'agit de nouvelles langues qui - bien que la très grande majorité de leur lexique soit d'origine française - ont des systèmes grammaticaux autonomes.

\subsection{Délimitation de la convergence - Rapports avec la copie et la congruence}

13 La convergence est une notion étroitement liée aux notions d'emprunt, de transfert ou de réplication. Kriegel et al. (2019) renoncent à ces termes en faveur de l'approche du code copying d'abord proposée par Johanson (2002, 2005, 2008). Cette décision est motivée par le fait que des traits morphosyntaxiques présents dans les langues créoles suite à des processus de convergence n'ont pas les mêmes propriétés que les structures dans les langues de départ. Si Johanson (2002) a choisi le terme de copying (copiage), il se réfère à la pratique médiévale des copistes qui ont copié des manuscrits à la main en laissant une place importante à leurs connaissances et leur arrière-plan culturels et pour qui le degré de fidélité à l'original n'était pas le principal enjeu. En ce sens, la métaphore de la copie est particulièrement adaptée aux processus de convergence décrits ici : ce sont des êtres humains (copistes ou locuteurs) qui, en effectuant une copie, la modifient selon leurs connaissances culturelles ou/et linguistiques. Johanson distingue des cas de global copying (copiage global) de différents cas de partial copying (copiage partiel). Dans Kriegel et al. (2019), nous distinguons essentiellement des copies ouvertes et des copies couvertes. Une copie ouverte reprend les aspects sonores, structuraux et sémantiques, cas très rare dans les créoles où nous rencontrons plus souvent des copies couvertes. Les copies couvertes reprennent seulement des aspects sémantiques et/ou structuraux sans copier la forme sonore. Ainsi, l'exemple de (f)in/'n discuté en 3. doit être considéré comme une copie couverte parce que les traits sonores ne sont pas concernés.

Quel est donc le rapport entre copie et convergence? La copie réfère au simple fait qu'un trait d'un code A est copié (intégralement ou partiellement) dans un code B, indépendamment $\mathrm{du}$ fait si $\mathrm{B}$ possède un trait qui est perçu comme similaire. Les raisons en sont diverses : il peut s'agir d'un élément particulièrement saillant, fréquent ou régulier. Pour ne citer que quelques exemples de "simples" copies sans convergence, on peut rappeler le vocatif '-o' en créole seychellois qui semble être un transfert direct du malgache (Bollée, 1982 : 76), le marquage du pluriel nominal dans les créoles haïtien, louisianais et guyanais par le pronom personnel de la $3^{\mathrm{e}}$ personne du pluriel yo/ye ainsi que le marquage du bénéficiaire d'une action par l'élément $b a(<\mathrm{fr}$. 'bailler') qui semblent être des transferts des langues ouest-africaines (Goodman, 1964 : 
46 et 63, Bollée, $1982: 72$ et 64). Dans la convergence, en revanche, une copie a lieu parce que les locuteurs perçoivent une similarité entre le trait dans A et dans B.

Un autre terme souvent utilisé dans la littérature est celui de congruence comme p. ex. dans l'approche de la compétition et de la sélection de Mufwene (p.ex. 2001, 2008) et plus récemment par Aboh (2015) qui formule un « principe de la congruence » :

The principle of congruence

Those morphosyntactic features that are (partly) shared by the superstrate and the substrate languages are more likely to be selected into the emerging creole than conflicting patterns. (Aboh, $2015: 63$ )

La principale différence entre notre terme de convergence et celui de la congruence consiste dans le fait que la congruence est un terme statique et purement descriptif qui renvoie à une proximité typologique et structurelle. La convergence, en revanche, est une notion dynamique, basée sur une activité discursive et cognitive du locuteur et favorisée par des conditions environnantes (Kriegel et al., 2019 : 325).

\section{Etude de cas : Résultat d'une double convergence - La marque du parfait dans les créoles mauricien et seychellois}

\subsection{Données des créoles mauricien et seychellois}

17 Fin(i) est le premier marqueur préverbal attesté en créole mauricien ${ }^{2}$. Il apparaît dans le tout premier échantillon d'une variété que l'on pourrait qualifier de " pré-créole » qui date de 1734, seulement treize ans après le début de la colonisation française de l'Ile de France, aujourd'hui Ile Maurice.

(1) créole mauricien ancien

\begin{tabular}{|l|l|l|}
\begin{tabular}{|l|l|}
$\begin{array}{l}\text { moy } \\
\text { 1SG }\end{array}$ & fini \\
PRF
\end{tabular} & $\begin{array}{l}\text { mouri } \\
\text { mourir }\end{array}$ \\
\hline 'Je suis mort.' (Chaudenson, $1981: 77)$ \\
\hline
\end{tabular}

18 A partir de 1822, fin apparaît avec d'autres verbes que 'mourir/mort' : Dans l'exemple (2), le verbe dynamique ‘donner' est précédé de fin.

(2) créole mauricien ancien

\begin{tabular}{|l|l|l|l|l|l|l|}
\hline $\begin{array}{l}\text { Moussié Sirandale } \\
\text { Monsieur Sirandale }\end{array}$ & $\begin{array}{l}\text { fin } \\
\text { PRF }\end{array}$ & $\begin{array}{l}\text { donne } \\
\text { donner }\end{array}$ & $\begin{array}{l}\text { nous } \\
\text { 1PL }\end{array}$ & $\begin{array}{l}\text { boire } \\
\text { boire }\end{array}$ & $\begin{array}{l}\text { zour } \\
\text { jour }\end{array}$ & $\begin{array}{l}\text { Noël } \\
\text { Noël }\end{array}$ \\
\hline \\
'Monsieur Sirandale nous a donné à boire le jour de Noël.' (Le Cernéen 1 janvier 1839, cité d'après \\
Baker, 2003:129)
\end{tabular}

19 Dans les variétés contemporaines du créole mauricien, le marqueur du parfait (perfect marker) ${ }^{3}$ fin est utilisé avec les variantes raccourcies in et 'n alors qu'en créole 
seychellois seulement les formes raccourcies in et ' $n$ sont courantes. Dans la présente contribution, je parle de (f)in/'n qui, dans les exemples concrets, se présente majoritairement sous la forme abrégée in ou ' $n$. Ce marqueur remontant à la périphrase verbale française 'finir de' est fortement grammaticalisé dans ces deux créoles et fait pleinement partie du paradigme des marques TMA. ${ }^{4}$

Pour le créole seychellois, Michaelis \& Rosalie (2013) constatent que le marqueur du parfait in/'n combiné avec des verbes dynamiques réfère à a past event with current relevance for the speech moment, e.g. zot in manze 'they have eaten'. Au sujet des verbes statifs et des adjectifs, ils précisent Some stative verbs receive a change of state interpretation when combined with in: mon konnen 'I know' vs. mon'n konnen 'I have come to know'. Adjectival verbs with in also refer to a change of state resulting from a past event: Son figir in rouz 'His face has become red.' Dans nos corpus de créole seychellois contemporain, les exemples avec des verbes aussi bien dynamiques que statiques sont très nombreux.

(3) créole seychellois

\begin{tabular}{|c|c|c|c|c|c|c|c|c|c|}
\hline $\begin{array}{l}\text { Ler } \\
\text { quand }\end{array}$ & $\begin{array}{l}\text { mon } \\
1 S G\end{array}$ & $\begin{array}{l}\text { 'n } \\
\text { PRF }\end{array}$ & $\begin{array}{l}\text { al } \\
\text { aller }\end{array}$ & $\begin{array}{l}\text { kot } \\
\text { chez }\end{array}$ & $\begin{array}{l}\text { dokter } \\
\text { docteur }\end{array}$ & $\begin{array}{l}\text { dokter } \\
\text { docteur }\end{array}$ & $\begin{array}{l}\text { pa } \\
\text { NEG }\end{array}$ & $\begin{array}{l}\text { 'n } \\
\text { PRF }\end{array}$ & $\begin{array}{l}\text { konpran } \\
\text { comprendre }\end{array}$ \\
\hline
\end{tabular}

21 Les exemples de l'emploi de in/' $n$ avec un adjectif qui se réfèrent à un changement d'état comme suite d'un procès passé sont plus rares mais également attestés : en (4), le locuteur explique l'état d'esprit d'adoucissement qu'il a atteint suite à la méditation et la prière.

\section{(4) créole seychellois}

\begin{tabular}{|l|l|l|l|l|l|l|l|l|l|l|l|l|}
\hline $\begin{array}{l}\text { apre } \\
\text { après }\end{array}$ & $\begin{array}{l}\text { mon } \\
1 \mathrm{SG}\end{array}$ & $\begin{array}{l}\text { 'n } \\
\text { PRF }\end{array}$ & $\begin{array}{l}\text { santi } \\
\text { sentir }\end{array}$ & $\begin{array}{l}\text { mwan } \\
1 \mathrm{SG} . \mathrm{OBJ}\end{array}$ & $\begin{array}{l}\text { mon } \\
1 \mathrm{SG}\end{array}$ & $\begin{array}{l}\text { 'n } \\
\mathrm{PRF}\end{array}$ & $\begin{array}{l}\text { dous } \\
\text { doux }\end{array}$ & $\begin{array}{l}\text { 'skan } \\
\text { jusqu'à }\end{array}$ & $\begin{array}{l}\text { mon } \\
1 \mathrm{SG}\end{array}$ & $\begin{array}{l}\text { n } \\
\text { PRF }\end{array}$ & $\begin{array}{l}\text { tro } \\
\text { trop }\end{array}$ & $\begin{array}{l}\text { dous } \\
\text { doux }\end{array}$ \\
\\
'Then I felt myself to have become soft until I have become too soft.' (Vidot-Rosalie corpus 2017) \\
'Après je me suis senti adouci jusqu'au point d'être devenu trop doux.'
\end{tabular}

Pour le créole mauricien, Baker \& Kriegel (2013) observent un comportement comparable du marqueur (f)in/'n pour les verbes dynamiques et les adjectifs ('adjectival verbs'). Véronique (2001: 40) souligne que fin n'est compatible principalement qu'avec les prédicats présentant le trait sémantique (+dynamique) ou avec certains statifs.

23 En (5), nous trouvons la forme ' $n n^{5}$ avec un verbe dynamique :

(5) créole mauricien

\begin{tabular}{|l|l|l|l|l|l|l|l|l|l|l|l|}
\hline $\begin{array}{l}\text { Mo } \\
\text { POSS }\end{array}$ & $\begin{array}{l}\text { Papa } \\
\text { papa }\end{array}$ & $\begin{array}{l}\text { sort } \\
\text { sortir }\end{array}$ & $\begin{array}{l}\text { depi } \\
\text { ABL }\end{array}$ & $\begin{array}{l}\text { Sesel, } \\
\text { Seychelles }\end{array}$ & $\begin{array}{l}\text { li } \\
\text { 3SG }\end{array}$ & $\begin{array}{l}\text { nn } \\
\text { PRF }\end{array}$ & $\begin{array}{l}\text { vini } \\
\text { venir }\end{array}$ & $\begin{array}{l}\text { pou } \\
\text { pour }\end{array}$ & $\begin{array}{l}\text { travay } \\
\text { travailler }\end{array}$ & $\begin{array}{l}\text { dan } \\
\text { LOC }\end{array}$ & $\begin{array}{l}\text { Moris } \\
\text { Maurice }\end{array}$ \\
\hline
\end{tabular}


'Mon père vient des Seychelles, il est venu pour travailler à Maurice (...).' (Bord la Mer 1980)

$$
\begin{aligned}
& \text { comme suite d'un procès passé. Dans cette conversation entre une vieille dame et sa } \\
& \text { voisine, des travaux d'agrandissement d'une terrasse ont mené au résultat que la } \\
& \text { terrasse est devenue jolie. }
\end{aligned}
$$

(6) créole mauricien

\begin{tabular}{|l|l|l|l|l|l|l|l|}
\hline \begin{tabular}{l|l} 
Li \\
3SG
\end{tabular} & $\begin{array}{l}\text { 'n } \\
\text { PRF }\end{array}$ & $\begin{array}{l}\text { zoli } \\
\text { joli }\end{array}$ & $\begin{array}{l}\text { aster-la, } \\
\text { maintenant }\end{array}$ & $\begin{array}{l}\text { en } \\
\text { ART }\end{array}$ & $\begin{array}{l}\text { gran } \\
\text { grand }\end{array}$ & $\begin{array}{l}\text { plas } \\
\text { place }\end{array}$ & $\begin{array}{l}\text { aster-la } \\
\text { maintenant }\end{array}$ \\
\hline
\end{tabular}

En français, la périphrase verbale 'finir de' est attestée depuis 1573 (FEW, https:// apps.atilf.fr/lecteurFEW/index.php/page/lire/e/115764). Malgré sa fréquence d'utilisation importante, son degré de grammaticalisation reste faible. Nous venons de voir qu'en créole mauricien et seychellois en revanche, (f)in/'n est devenu une marque aspectuelle fortement grammaticalisée qui s'insère pleinement dans le paradigme des marqueurs TMA les plus fréquemment employés (voir aussi Véronique 2001). Déjà Bickerton (1981 : 94) constate que la présence de la marque (f)in/' $n$ dans les créoles mauricien et seychellois "dérange " le système TMA prototypique des créoles et se demande pourquoi ces créoles have let loose their completives.

\subsection{Les successeurs de 'finir de' dans les autres créoles français}

Certes, les successeurs de la périphrase verbale 'finir de' sont également attestés dans les autres créoles français. Cependant, ils restent souvent proches du français et n'ont guère été soumis au processus de grammaticalisation qu'ils ont parcouru dans les créoles mauricien et seychellois. Souvent, fin est qualifié d'auxiliaire (NeumannHolzschuh, 1985: 234 pour le créole louisianais; Valdman, 2015: 228 pour le créole haïtien, Watbled, 2015 pour le créole réunionnais), mais pas comme marque TMA. Pour le créole guadeloupéen, le dictionnaire de Ludwig et al. (2002) indique sous l'entrée fin/ fini des valeurs sémantiques qui ne laissent pas penser à un processus de grammaticalisation avancé. Cependant, pour le créole martiniquais, Bernabé $(2003,148)$ parle de passé récent et remarque : Cet aspect est exprimé par le morphème fini. ${ }^{6}$ Pour le créole guyanais, Pfänder (2000: 201) dans une étude exhaustive des systèmes TMA mentionne brièvement que fin(i) peut assumer, dans certaines variétés de ce créole, une valeur d'egressif. Le seul créole de la zone américano-caraïbe où la marque est employée plus amplement est le créole haïtien mais son emploi y est plus limité qu'en créole mauricien et seychellois (Fattier, 2013 ; Valdman, 2015). Après une discussion de l'évolution de (f)in/'n dans l'océan Indien, Baker (2003: 131) conclut: Aucun usage comparable d'un marqueur dérivé de finir semble avoir été rapporté en créole antillais ou en créole guyanais. En ce qui concerne le créole réunionnais, l'emploi de fin est marginal étant donné que le parfait est normalement exprimé par la particule la. Après avoir 
donné des exemples dans lesquels la et fin sont combinés, Watbled (2015) constate à l'égard de fin:

Cette forme /fin(i)/ exprime l'existence de l'occurrence de l'événement dans une période ayant commencé avant un repère (le moment de l'énonciation dans les deux exemples ci-dessus), en même temps que le positionnement mental de l'énonciateur au-delà de la borne droite de l'événement. (Watbled, 2015) .

Une analyse comparative plus approfondie concernant le rôle de fin dans tous les créoles français dépasserait le cadre de cet article. Cependant, même si une analyse quantitative reste à faire, il semble évident que les emplois de la marque sont beaucoup moins fréquents dans les autres créoles français que dans les créoles mauricien et seychellois.

\subsection{Données des langues serviles - Ile Maurice et Seychelles}

Avant de proposer une interprétation de la forte grammaticalisation de (f)in/'n en créole mauricien et en créole seychellois, quelques éléments sociohistoriques concernant les migrations de populations dans l'océan Indien de l'ouest sont nécessaires.

L'Ile Maurice est colonisée par la France à partir de 1721. Avec l'essor de l'industrie de la canne à sucre, les colons importent des populations serviles de différents endroits mais dès le départ Madagascar joue un rôle important pour ensuite progressivement céder sa place à l'Afrique de l'Est.

In the thirty years from 1735, Madagascar was the main source of slaves, but slaves speaking Bantu languages had begun to be introduced from 1736 and their proportion slowly increased until they overtook Malagasy arrivals decisively in about 1765. (Baker \& Kriegel, 2013)

L'existence d'une langue créole est attestée dès 1773 , donc seulement une cinquantaine d'années après le début de la colonisation. Les Seychelles sont peuplées essentiellement par des colons mauriciens et leurs esclaves à partir de 1770 et le créole seychellois est souvent considéré comme une prolongation du créole mauricien.

$\mathrm{Au} \mathrm{vu}$ de ces faits démographiques, il convient de regarder des données de deux groupes de langues pour savoir s'il est possible d'envisager une interprétation liée aux situations de contact de langues : le malgache d'un côté, langue parlée par la majorité de la population servile jusqu'à dans les années 1760 et diverses langues bantoues de l'Est, langues parlées par une majorité d'esclaves après les années 1760 de l'autre. L'idée que l'évolution de (f)in/'n pourrait être liée à une, voire des influences substratiques n'est pas nouvelle. Ainsi, Adam (1883) évoque une possible influence du malgache, hypothèse réfutée avec véhémence par Chaudenson (2003: 354). Corne (1982: 101) mentionne des modèles éventuels des langues bantoues de l'Est pour discuter, dans un article de 1983, une possible influence conjointe des langues bantoues de l'Est et du malgache ${ }^{8}$.

\subsubsection{Le malgache}

Comme illustré avec l'exemple (1), la présence de fin(i) est déjà attestée dans le tout premier témoignage de la phase de formation du créole mauricien datant de 1734 . A cette date, on peut supposer que la population servile était composée d'une majorité de 
locuteurs du malgache. Adam (1883) est le premier à proposer un lien entre l'emploi de fin et une particule présente en malgache.

Le créole possède un passé absolu formé par la préposition au thème verbal de fine « fini », et un plus-que-parfait formé par la préposition des deux particules té et fine. Ex. : mo fine manzé j'ai mangé, j'ai fini de manger; mo té fine manzé j'avais mangé, j'eus mangé ; mo fine fini j'ai fini. (...)

Malgache. - «Efa est un participe-racine qui signifie « fini ». Ce mot est très usité...Il sert à former le plus-que-parfait : efa ni-ambina alina j'avais fini de veiller (...) L'emploi de fine comme indice temporal a été suggéré aux nègres par le souvenir du malgache efa. (Adam, 1883: 65, https://gallica.bnf.fr/ark:/12148/bpt6k74263t/ f65.item.texteImage.zoom)

Dans sa grammaire de 1964, Rajemisa-Raolison (1964: 78) range efa dans les «particules modificatives des temps » et note, tout comme le fait déjà Adam, qu'il s'agit d'une marque très fréquente et polyvalente en malgache.

\section{(7) malgache}

\begin{tabular}{|l|l|}
\hline Efa. & $\begin{array}{l}\text { - Devant le présent, indique qu'on est déjà en train de... } \\
\text { Ex. Efa mihinana izy (il est déjà en train de manger) }\end{array}$ \\
\hline & $\begin{array}{l}\text { - Devant le passé, indique qu'on a fini de ... } \\
\text { Ex. Efa nihinana izy (il a fini de manger) }\end{array}$ \\
\hline $\begin{array}{l}\text { - Devant le futur, indique qu'on est sur le point de... } \\
\text { Ex. Efa hihinana izy (il est sur le point de manger) }\end{array}$ \\
\hline (Rajemisa-Raolison, 1964: 78)
\end{tabular}

34 La possibilité d'établir une équivalence entre (f)in/' $n$ et efa devant une forme du passé s'impose d'autant plus si on tient compte du fait qu'efa doit se traduire par "achevé » (Rahajarizafy, $1960: 29$, voir aussi Adam 1883 ci-dessus qui traduit par « fini »).

Dans Rahajarizafy (1960 : 149), l'exemple suivant permet de mener plus loin le parallèle avec les créoles mauricien et seychellois :

\section{(8) malgache}

\begin{tabular}{|c|c|c|c|}
\hline Efa & afaka & izao & Rakoto \\
\hline PRF & libéré & maintenant & Rakoto (glosage par S.K.) \\
\hline
\end{tabular}

Cet exemple montre qu'efa est également utilisé devant des adjectifs qui résultent d'un procès comme c'est le cas de (f)in/' $n$ dans les créoles seychellois et mauricien (exemples (4) et (6)). 


\subsubsection{Les langues bantoues de l'Est}

Dans sa discussion de modèles présents dans les langues bantoues de l'Est, Corne (1983) donne l'exemple suivant dans la catégorie des verbes dynamiques :

(9) makua

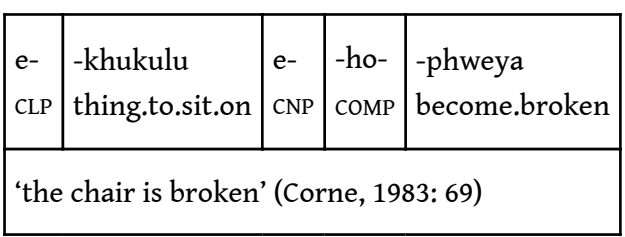

qui correspond en créole mauricien à « sez fin kase » ('La chaise s'est cassée/est cassée') (Corne, 1983). Dans la catégorie des adjectifs qu'il appelle "processive predicates », il donne l'exemple suivant traduit dans plusieurs langues bantoues qui utilisent des marques différentes mais équivalentes d'un point de vue sémantique :

(10) exemples bantoues de Corne $(1983: 69)$

\begin{tabular}{|l|l|l|}
\hline (Shona) & nda- & - neta \\
\hline & PAS.I & become.tired \\
\hline
\end{tabular}

\begin{tabular}{|l|l|l|l|}
\hline $\begin{array}{l}\text { (Makuwa) } \\
\text { (Swahili) } \\
(\text { IdeFC })^{9}\end{array}$ & $\begin{array}{l}\text { ni- } \\
\text { mo }\end{array}$ & $\begin{array}{l}\text {-ho- } \\
\text { fin }\end{array}$ & $\begin{array}{l}\text {-chiya } \\
\text {-choka } \\
\text { fatige }\end{array}$ \\
\hline & I & comP & become.tired \\
\hline
\end{tabular}

Selon Michaelis \& Rosalie (2013), le makua et le swahili comptent parmi les langues de substrat les plus probables dans la genèse des créoles mauricien et seychellois. La description que donne Woodward (1926: 289) de la marque ho du makua rappelle ce que nous observons pour les créoles seychellois et mauricien où in/'n permet d'insister sur le résultat précédé par un procès.

The Present Perfect tense is made by prefixing -ho- to the verb. (...) In verbs denoting a state or the possession of a quality, the present has the meaning of to enter upon, to acquire or become what the verb denotes. The -ho- tense must then be translated by "to have", or "to be" etc.

chinonta, it is getting putrid

chihonta, it is putrid

(Woodward, $1926: 289$ )

L'exemple donné par Woodward correspond non seulement aux exemples cités par Corne mais aussi à nos données de corpus (4) et (6) du créole seychellois et mauricien où (f)in/'n est utilisé avec des adjectifs. Notons que la similarité n'est pas seulement sémantique mais également syntaxique : Aussi bien pour le swahili que pour le makua que pour nos données créoles il s'agit d'une marque préposée au verbe. 


\section{Conclusion - Convergences}

41 Je propose d'interpréter les données présentées en 3.3 comme étant le résultat d'une double convergence : la tendance de grammaticalisation de la périphrase verbale 'finir de' présente en français a été continuée dans les créoles mauricien et seychellois. Cette continuation peut s'expliquer par les langues premières des apprenants qui ont servi en quelque sorte de catalyseur: Pendant la phase de créolisation au $18^{\mathrm{e}}$ siècle les apprenants du français ont perçu des similarités sémantiques et syntaxiques entre les marques existantes dans leurs langues avec la périphrase 'finir de' du français. Bien évidemment, des recherches plus poussées sur la sémantique précise des marqueurs malgaches et p.ex. makua sont nécessaires. Cependant, la notion de "similarité perçue" permet d'appuyer notre hypothèse de convergence. Même si la sémantique et les contextes d'emploi des marques ho (macua), efa (malgache) et de la périphrase verbale 'finir de' du français ne sont sûrement que partiellement congruents nous interprétons la grammaticalisation de (f)in/'n comme le résultat d'une convergence. Son emploi limité dans les créoles français de la Caraïbe permet d'appuyer cette hypothèse. Nos données soutiennent fortement la suggestion de Corne (1983) qui conclut que a given phenomenon in a Creole language may arise from multiple converging influences.

Il est évident que l'évolution des langues créoles s'explique par une multitude de facteurs écologiques divers. La présente contribution vise à montrer que la convergence est un de ces facteurs dont le rôle ne doit pas être sous-estimé.

\section{BIBLIOGRAPHIE}

Aboh, E.O. (2015) The Emergence of Hybrid Grammars. Language Contact and Change, Cambridge, UK: Cambridge University Press (Cambridge Approaches to Language Contact).

Adam, L. (1883) Les idiomes négro-aryen et maléo-aryen, Paris.

Baker, P. \& C. Corne (1982) Isle de France Creole. Affinities and Origins, Ann Arbor.

Baker, P. (2003) Quelques cas de réanalyse et de grammaticalisation dans l'évolution du créole mauricien, in Kriegel, S. (ed.) Grammaticalisation et réanalyse. Approches de la variation créole et française, Paris: CNRS Editions, Langage, p. 111-142.

Baker, P. \& S. Kriegel (2013) Mauritian Creole, in Michaelis, S.M., Maurer, P., Haspelmath, M. \& M. Huber (eds) The survey of pidgin and creole languages. Volume 2: Portuguese-based, Spanish-based, and French-based Languages, Oxford: Oxford University Press.

Baptista, M., Gelman, S. \& E. Beck (2016) Testing the role of convergence in language acquisition, with implications for creole genesis, International Journal of Bilingualism, 20, 3, p. 269-296.

Bernabé, J. (2003) Précis de syntaxe créole, Guyane. Martinique. Guadeloupe. Paris. Réunion: Ibis Rouge Editions. 
Bollée, A. (1982) Die Rolle der Konvergenz bei der Kreolisierung, in Ureland, P.S. (ed.) Die Leistung der Strataforschung und der Kreolistik. Typologische Aspekte der Sprachkontakte. Akten des 5. Symposions über Sprachkontakt in Europa. Mannheim 1982, 1982, Tübingen: Niemeyer, p. 391-405.

Bickerton, D. (1981) Roots of Language, Ann Arbor: Karoma.

Bruyn, A. (2009) Grammaticalization in creoles: Ordinary and not-so-ordinary cases, Studies in Language, 33, 2, p. 312-337.

Chaudenson, R. (1981) Textes créoles anciens (La Réunion et Ile Maurice). Comparaison et essai d'analyse, Hamburg: Helmut Buske Verlag (Kreolische Bibliothek, 1).

Chaudenson, R. (2003) La créolisation : théorie, applications, implications, Paris: L'Harmattan.

Corne, C. (1982) A contrastive Analysis of Reunion and Isle de France Creole French: Two Typologically Diverse languages, in Baker, P. \& C. Corne Isle de France Creole. Affinities and Origins, Ann Arbor: Karoma, p. 8-130.

Corne, C. (1983) Substratal reflections: The completive aspect and the distributive numerals in Isle de France Creole, Te Reo, p. 65-80.

Fattier, D. (2013) Haitian Creole, in Michaelis, S.M., Maurer, P., Haspelmath, M. \& M. Huber (eds) The survey of pidgin and creole languages. Volume 2. Portuguese-based, Spanish-based, and French-based Languages, Oxford: Oxford University Press.

FEW = Wartburg, Walther von, et al. (1928-2003) Französisches Etymologisches Wörterbuch. Eine Darstellung des galloromanischen Sprachschatzes [en ligne], 3, Bonn: Klopp ; Bâle: Zbinden, p. 556-560. Disponible sur : https://apps.atilf.fr/lecteurFEW/index.php (consulté le 10 novembre 2020).

Goodman, M.F. (1964) A comparative study of Creole French dialects, The Hague: Mouton.

Gumperz, J.J. \& R. Wilson (1971) Convergence and creolization. A case from the Indo-Aryan/ Dravidian border in India, in Dil, A.S. (ed.) Language in social groups. Essays by John J. Gumperz, Stanford: Stanford University Press, p. 251-273.

Heine, B. \& T. Kuteva (2005) Language contact and grammatical change, Cambridge, UK: Cambridge University Press.

Hilty, G. (1975) Westfränkische Superstrateinflüsse auf die galloromanische Syntax, Romanische Forschungen, 87, p. 413-426.

Jarvis, S. \& A. Pavlenko (2008) Crosslinguistic influence in language and cognition. New York. London: Routledge.

Johanson, L. (2002) Contact-induced change in a code-copying framework, in Jones, M.C. \& E. Esch (eds.) Language change. The interplay of internal, external and extra-linguistic factors, Berlin. New York: De Gruyter, p. 85-313.

Johanson, L. (2005) On copying grammatical meaning, Sprachtypologie und Universalienforschung, 58 , p. $75-83$.

Johanson, L. (2008) Remodeling grammar. Copying, conventionalization, grammaticalization, in Siemund, P. \& N. Kintana (eds) Language contact and contact languages, Amsterdam. Philadelphia: Benjamins, p. 61-79.

Kihm, A. (1988) Conflation as a directive process in creolization, in Boretzky, N., Enninger, W. \& T. Stolz (eds) Beiträge zum 4. Essener Kolloquium über 'Sprachkontakt, Sprachwandel, Sprachwechsel, Sprachtod' 1987, Bochum: Brockmeyer, p. 111-137. 
Kriegel, S. (1996) Diathesen im Mauritius- und Seychellenkreol, Tübingen: Gunter Narr, ScriptOralia.

Kriegel, S. (2003) Introduction : Vers une interprétation multicausale du changement linguistique, in Kriegel, S. (ed.) Grammaticalisation et réanalyse: Approches de la variation créole et française, Paris: CNRS Editions (Langage).

Kriegel, S. (2003) (ed.) Grammaticalisation et réanalyse : Approches de la variation créole et française, Paris: CNRS Editions (Langage).

Kriegel, Sibylle (2021) Grammaticalization in Seychelles Creole: the coding of reciprocity by kanmarad [en ligne], Isogloss. Open Journal of Romance Linguistics, 7, 11, p. 1-27. Disponible sur : https://doi.org/10.5565/rev/isogloss.149 (consulté le 10 septembre 2021).

Kriegel, S., Ludwig, R. \& S. Pfänder (2019) Dialectes - créolisation - convergence. Quelques hypothèses à partir du berrichon et du poitevin-saintongeais, in Dufter, A., Grübl, K. \& T. Scharinger (eds) Des parlers d'oil à la francophonie: contact, variation et changement linguistique, Berlin: De Gruyter, supplément, p. 299-347.

Lehmann, C. (1995) Thoughts on Grammaticalization, München: Lincom Europa, $1^{\text {re }}$ pub. 1982, Cologne: University of Cologne (AKUP 48).

Ludwig, R., Montbrand, D., Poullet, H. \& S. Telchid (eds) (2002) Dictionnaire créole, 2e éd. révisée, Paris: SERVEDIT, Maisonneuve \& Larose, Éditions Jasor.

Ludwig, R., Mühlhäusler, P. \& S. Pagel (2019) Linguistic ecology and language contact: Conceptual Evolution, Interrelatedness, and Parameters, in Ludwig, R., Mühlhäusler, P. \& S. Pagel (eds.) Linguistic ecology and language contact, Cambridge, UK: Cambridge University Press (Cambridge Approaches to Language Contact), p. 3-42.

Michaelis, S. (1993) Temps et aspect en créole seychellois : valeurs et interférences, Hamburg: Helmut Buske (Kreolische Bibliothek, 11).

Michaelis, S.M. \& M. Rosalie (2013) Seychelles Creole, in Michaelis, S.M., Maurer, P. \&, Haspelmath, M. \& M. Huber (eds) The survey of pidgin and creole languages. Volume 2: Portuguesebased, Spanish-based, and French-based Languages, Oxford: Oxford University Press.

Mufwene, S.S. (2001) The ecology of language evolution, Cambridge, UK: Cambridge University Press. Mufwene, S.S. (2008) Language evolution: Contact, competition and change, London: Continuum. Neumann-Holzschuh, I. (1985) Le créole de Breaux Bridge, Louisiane. Etude morphosyntaxique - textes vocabulaire, Hamburg: Helmut Buske Verlag (Kreolische Bibliothek, 7).

Pfänder, S. (2000) Aspekt und Tempus im Frankokreol. Tübingen: Gunter Narr, ScriptOralia.

Rahajarizafy, A. (1960) Essai sur la grammaire malgache, Antanimena Tananarive: Imprimerie catholique.

Rajemisa-Raolison, R. (1964) Grammaire malgache, Fianarantsoa: Centre de formation pédagogique.

Schuchardt, H. (1900) Über die Klassifikation der romanischen Mundarten [en ligne], in Rassedenken in der Sprach- und Textreflexion, Graz: K.u.K. Buchdruckerei Styria. Disponible sur : https://doi.org/10.30965/9783846758762_028 (consulté le 8 juillet 2021).

Valdman, A. (2015) Haitian Creole. Structure, variation, status, origin, Sheffield: Equinox.

Véronique, D.G. (2001) Temps, aspect et mode en mauricien, L'Information grammaticale, 89, p. 38-42. 
Watbled, J.-P. (2015) Les particularités morphosyntaxiques du créole réunionnais, Études Créoles Vol. XXXIII $n^{\circ} 2$ - 2015 [en ligne]. Disponible sur : http://www.lpl-aix.fr/ fulltext/Etudes_Creoles/ watbled.pdf (consulté le 15 mai 2021).

Weinreich, U. (1953) Languages in Contact. Findings and Problems, New York: Linguistic Circle.

Woodward, H.W. (1926) An outline of Makua grammar, Bantu Studies 2, p. 269-325.

\section{ANNEXES}

Corpus:

Anonyme (1980) Bord la mer, Port Louis : Port Louis Harbour and Dock Workers Union, Maurice.

Vidot-Rosalie (2017) Corpus oral en collaboration avec Marcel Rosalie, Seychelles.

\section{NOTES}

1. Nous n'excluons en aucun cas l'existence de processus de grammaticalisation "classiques» internes à la langue qui ne sont pas motivés, déclenchés ou même renforcés par les langues en contact comme c'est p.ex. le cas du marquage de la réciprocité en créole seychellois (Kriegel, 2021).

2. Pour une discussion de l'alternance forme longue et forme courte et plus d'exemples de textes anciens voir Baker (2003: 128).

3. La terminologie étant hétérogène, j'utiliserai celle proposée par APiCS qui opte pour «perfect marker », marqueur du parfait. D'autres travaux, comme p.ex. Corne (1983) parlent de marque du complétif.

4. Pour une étude détaillée de son emploi en créole seychellois voir Michaelis (1993). Pour une présentation des marques TMA du créole mauricien voir Véronique (2001).

5. Nous gardons les graphies originales des exemples.

6. Je souhaiterais remercier Bohdana Librova pour ces remarques sur les créoles antillais.

7. Je souhaiterais remercier Daniel Véronique pour cette référence bibliographique et son analyse de la situation en créole réunionnais. Il constate que fin n'y connaît effectivement pas le même usage que dans les deux autres créoles de la zone et propose une explication qui établit un lien entre ce fait et la longue phase de la période d'habitation à la Réunion (1680-1725) qui différencie cette île de l'Ile de France (Ile Maurice). Des peuplements identiques, dans les mêmes proportions, produisent des effets linguistiques différents car d'autres circonstances socio-économiques façonnent différemment les espaces sociaux. (Daniel Véronique, p.c.)

8. Il semblerait que Corne ne connaissait pas le travail d'Adam (1883).

9. IdeFC est le sigle pour Ile de France Créole qui comprend les créoles mauricien et seychellois dans la terminologie utilisée par Corne. 


\section{RÉSUMÉS}

Après un bref historique de l'utilisation de la notion de convergence essentiellement en études romanes et créoles (1.), je présenterai l'approche proposée par Kriegel et al. (2019) et je limiterai la convergence par rapport à d'autres notions centrales en linguistique de contact et en études créoles (2.). Ensuite, la contribution présentera une étude de cas (3.) : la marque du parfait (f)in/'n dans les créoles mauricien et seychellois. Il s'agit certes de « matériaux de construction » français parce qu'on y reconnaît la périphrase verbale française 'finir de'. Cependant, le degré de grammaticalisation de (f)in/'n dans les créoles mauricien et seychellois est très élevé étant donné que cette marque fait partie du paradigme des particules TMA essentielles. Il n'en est pas de même dans les créoles français de la zone américano-caraïbe où la périphrase verbale 'finir de' a laissé des traces sans pour autant avoir connu une grammaticalisation poussée. Contrairement à Kriegel et al. (2019) qui présente un cas de convergence (la marque du réfléchi) en mettant l'accent sur la continuité avec les dialectes français, la présente contribution présente un cas de convergence (la marque du parfait) en insistant sur le rôle des langues parlées par les populations serviles dans l'océan Indien. Je montrerai que ces langues ont joué un rôle essentiel de catalyseur pour la grammaticalisation de (f)in/' $n$. L'interaction entre les langues en présence est d'autant plus remarquable qu'il s'agit de langues appartenant à des familles très différentes : d'un côté, les langues bantoues de l'Est et de l'autre côté le malgache, langue austronésienne. Dans le domaine de l'expression du parfait (ou de la complétivité), les locuteurs malgaches, puis les locuteurs de langues bantoues de l'Est auraient perçu des similarités (Jarvis \& Pavlenko, 2008) entre leur langue première et la langue cible de leur apprentissage, le français en train de se transformer en créole au cours du $18^{\mathrm{e}}$ siècle. Pour l'évolution de la marque (f)in/'n, je propose donc de parler de double convergence dans l'évolution du français vers le créole mauricien, convergence avec le malgache et avec les langues bantoues de l'Est parlées par les populations serviles.

This contribution aims at a better understanding of the emergence of creole languages by examining the role of convergence in the evolution of the perfect marker (f)in/'n in Mauritian and Seychelles Creoles. Section 1 gives a brief historical outline of the use of the notion of convergence in Romance and in Creole studies. After a short discussion of early work e. g. by Gumperz \& Wilson (1971), it introduces the first precise definition of the notion given by Bollée (1982) in the context of Creole studies: according to her, convergence characterizes the phenomenon

(...) that similar structures of two languages in contact meet and reinforce one another, or that a tendency observable in one language becomes grammaticalised through the influence of a structure existing in the other. (Bollée, 1982: 392, my translation)

Building on Bollée's findings, I will then present the approach proposed by Kriegel et al. (2019) which develops the notions of similarity and grammaticalization. Language learners might perceive similarities between linguistic codes where linguists might not see them at first sight. In other words, convergence is a function of speakers who rely on perceiving similarities.

A perceived similarity is a conscious or unconscious judgment that a form, structure, meaning, function, or pattern that an L2 user has encountered in the input of the recipient language is similar to a corresponding feature of the source language. (Jarvis \& Pavlenko, 2008: 179)

As to the second important point in Bollée's definition, Kriegel et al. (2019) propose an extended notion of grammaticalization, traditionally used in a monolingual frame (e.g. Lehmann, 1995) and consider it in the plurilingual ecologies creole languages use to evolve in (e.g. Bruyn, 2009; Heine \& Kuteva, 2005; Kriegel, 2003).

TIPA. Travaux interdisciplinaires sur la parole et le langage, 37 | 2021 
In section 2, I will distinguish convergence from other central notions used in contact linguistics and in Creole studies: first, convergence processes are often described in terms of borrowing, replication, or transfer. Instead of using this terminology, Kriegel et al. (2019) work with an elaborated version of Johanson's $(2002,2005,2008)$ notion of code copying. The term copy simply refers to the fact that a feature in code A is copied (entirely or partially) into code B, independently from the question of perceived similarity. In convergence, the copy takes place because the speakers (learners) perceive a similarity between a feature in code A and in code B. Second, I refer to congruence as a static notion opposed to convergence as a dynamic one.

The paper will then present a case study (section 3): the perfect marker (f)in/' $n$ in Mauritian and in Seychelles Creoles. Undoubtedly the "construction material" is French because it is possible to distinguish the French verbal periphrasis 'finir de'. However, the grammaticalization of ( $f$ ) in/'n in Mauritian and in Seychelles Creoles is very advanced because the marker belongs to the closed set of the core TMA markers. This is not the case in the French creoles of the Caribbean where the verbal periphrasis 'finir de' left its traces but did not reach an advanced degree of grammaticalization. Contrary to Kriegel et al. (2019), which studies a case of convergence (the body reflexive) by focusing on the continuity with the French dialects spoken by the colonizers, the present contribution deals with a case of convergence (the perfect marker (f)in/'n) by insisting on the crucial role of the languages spoken by the enslaved populations in the Indian Ocean. I will show that these languages have played an essential catalytic role in the grammaticalization of (f)in/'n. This interaction between the languages in contact is all the more noteworthy if we consider that they belong to very different language families: on the one hand we have Eastern Bantu languages, on the other Malagasy, an Austronesian language. Concerning the expression of the perfect (or completivity) speakers of Malagasy, then speakers of Eastern Bantu languages would have perceived similarities (Jarvis \& Pavlenko, 2008) between their first language and the target language, the French variety on its way to becoming a Creole during the $18^{\text {th }}$ century. As to the evolution of the marker ( $f$ )in/' $n$, I suggest speaking of a double convergence during the evolution from French to Mauritian Creole, a convergence with Malagasy and with the Eastern Bantu languages spoken by the slaves.

\section{INDEX}

Mots-clés : convergence, créole mauricien, créole seychellois, grammaticalisation, parfait, similarité perçue

Keywords : convergence, grammaticalization, perceived similarity, perfect, Mauritian Creole, Seychelles Creole

\section{AUTEUR}

\section{SIBYLLE KRIEGEL}

Aix Marseille Univ, CNRS, LPL, Aix-en-Provence, France

sibylle.kriegel@univ-amu.fr 